\title{
PAPEL DA BRINQUEDOTECA NA RECUPERAÇÃO DA CRIANÇA HOSPITALIZADA SOB A ÓTICA DE PAIS E RESPONSÁVEIS
}

\author{
Fernanda Amorim Cesário1, Suelen Fernanda Coutinho Pinto1, Thaís de Freitas Aniceto1, \\ Alessandra Silva Lima Jardim1, Claudirene Milagres Araújo1 e Lilian Machado Torres² \\ ${ }^{1}$ Faculdade Ciências Médicas de Minas Gerais, Brasil. feamorim.sz@hotmail.com; suelen.coutinhobh@gmail.com; \\ thaisdefreitasa@gmail.com; aleenf@gmail.com; claudirene_milagres@hotmail.com \\ ${ }^{2}$ Hospital Governador Israel Pinheiro, Minas Gerais, Brasil. lilian.torres0806@gmail.com
}

\begin{abstract}
Resumo. Introdução: a hospitalização infantil representa incontáveis consequências para a criança, sua família e toda a equipe de saúde, concebidas por comportamentos depressivos e/ou agressivos e atitudes pouco colaborativas que interferem no cuidado em saúde. A brinquetoteca é um dos métodos para a humanização da assistência pediátrica. Objetivo: compreender como pais e responsáveis percebem a brinquedoteca na recuperação de suas crianças hospitalizadas. Método: pesquisa exploratória, descritiva, com abordagem qualitativa. Participaram 15 pais ou responsáveis pelas crianças internadas na unidade pediátrica, por meio de entrevistas, a partir de um roteiro semiestruturado. Os conteúdos dos depoimentos foram organizados e analisados pelo referencial de Bardin. Resultados: a sala de brincar é terapêutica e pais e responsáveis percebem a redução das tensões em suas crianças hospitalizadas, o aumento de sua autoestima e maior sensação de segurança, o que contribui para sua recuperação. Emergiram tres categorias temáticas: O tempo de hospitalização sendo ocupado; O vínculo criado pelas brincadeiras entre os responsáveis e as criança; e $\mathrm{O}$ brincar como recurso terapêutico. Conclusões: atividades lúdicas revestem-se da dimensão terapêutica, reconhecida pelos pais ou responsáveis. As equipes de saúde devem refletir e implementar brincadeiras que promovam atendimento humanizado e viabilizem maior relacionamento entre os pequenos e os adultos.
\end{abstract}

Palavras-chave: Enfermagem Pediátrica; Humanização da Assistência; Ludoterapia; Pais; Saúde da Criança Institucionalizada.

\section{PERCEPTION OF PARENTS OR GUARDIANS ABOUT THE TOY LIBRARY AS A THERAPEUTIC RESOURCE IN CHILD CARE}

\begin{abstract}
Introduction: the childhood hospitalization has countless consequences for the child, his family and the entire health team, which contribute to depressive and / or aggressive behaviours and, also, uncooperative attitudes that interfere in health care. The toy library is one of the methods for the humanization of paediatric care. Goals: to understand how parents and guardians perceive the toy library in the recovery of their hospitalized children. Method: exploratory, descriptive research with a qualitative approach. 15 parents or guardians of the children admitted to the paediatric unit participated, through an interview based on a semi-structured script. The results were organized and analysed by the Bardin framework. Results: the playroom is therapeutic and parents and guardians perceive the reduction of tensions in their hospitalized children, the increase in their self-esteem and a sense of security, which contributes to their recovery. Three thematic categories emerged: Hospitalization time being occupied; The bond created by games between parents and children; and Playing as a therapeutic resource. Conclusions: the playful activities have the therapeutic dimension, which was recognized by the parents or guardians. Health teams must reflect and implement more games that promote humanized care and are able to strengthen the bond between children and adults.
\end{abstract}

Keywords: Pediatric Nursing; Humanization of Assistance; Play Therapy; Parents; Health of Institutionalized Children. 


\section{INTRODUÇÃO}

Durante o processo de internação a criança pode apresentar vários sentimentos desagradáveis, devido à falta de maturidade para compreender seu estado de saúde, bem como pelo fato de estar em um ambiente desconhecido e afastada do apoio de seus familiares e amigos. Sofre, portanto, influências negativas que envolvem duas esferas: a da própria doença, mediante o processo de adoecimento e a do agravamento do seu estado psicológico. Tal experiência tem potencial para fazer emergir sentimentos de ansiedade, medo, abandono e culpa, e isso pode levá-la a comportamentos depressivos ou agressivos. Como consequências, advém as atitudes de pouca colaboração que dificultam as intervenções e os cuidados de Enfermagem. Trata-se de uma experiência traumática, não somente para crianças mas, para a família, também, (Gabatz, Terra, Couto, Milbrath, \& Schwartz, 2017; Leite, Vergílio, \& Silva, 2017; Gillard, 2019; Nurwulansari, Ashar, Huriati, \& Syarif, 2019).

Nessa faixa etária, muitas vezes, por não compreenderem a doença e a necessidade de hospitalização, as alterações em sua rotina representam a perda do controle sobre o próprio corpo. Entretanto, frente ao cuidado humanizado e acolhedor a vivência de tais sentimentos negativos podem ser minimizados, quando se estabelecem estratégias de comunicação adaptadas, que utilizam linguagem verbal e não verbal e que consideram as especificidades dos envolvidos (Gabatz, Terra, Couto, Milbrath, \& Schwartz, 2017).

Um dos métodos comuns para humanizar a assistência pediátrica inclui o lúdico que busca inserir o envolvido no funcionamento hospitalar e evidenciar sua dignidade. Por isso, o método tem sua aplicabilidade como recurso terapêutico positivo no cuidado à criança hospitalizada e cada vez mais vem sendo estudado (Araújo, Ribeiro, Sobral, Silva, \& Faro, 2017; Gillard, 2019; Nurwulansari, Ashar, Huriati, \& Syarif, 2019), a partir da avaliação das demandas infantis e dos familiares. São estratégias de entretenimento e de aproximação o brinquedo terapêutico e a pet terapia (Pinto, Andrade, Medeiros, Santos, Queiroz, \& Jales, 2015; Gabatz, Terra, Couto, Milbrath, \& Schwartz, 2017).

Além do prazer proporcionado pelas atividades lúdicas, no mundo infantil o método concorre para o desenvolvimento intelectual e social da criança, que inclui racionalizar, imaginar, interpretar e criar (Conceição, 2016). Assim, o processo de internação pediátrica acontece de forma positiva, o que otimiza o tratamento instituído (Silva \& Brandão, 2017). Ademais, a brinquedoteca constitui-se uma estrutura favorável, no ambiente hospitalar durante a 
internação, pois representa um espaço de bem-estar e acolhimento da criança que possibilita exposição de seus anseios, vivências e sentimentos envolvidos. Cria uma atmosfera que estimula a colaboração, além de promover maior tranquilidade e adaptação hospitalar (Marcolin, Marcolin, Souza, \& Barbosa, 2016).

Torna-se imprescindível compreender se, na perspectiva dos pais ou responsáveis, há a percepção do papel da brinquedoteca na recuperação de crianças hospitalizadas, considerando que já se comprovou sua eficácia na diminuição da ansiedade e do tempo de internação, além do fortalecimento de vínculos familiares. Tal compreensão poderá influenciar a relação dos profissionais com os pais ou responsáveis no incentivo às brincadeiras e solidificar mais brinquedotecas nos hospitais. As questões de investigação, portanto, pertinentes ao objeto de estudo, foram assim definidas: brincar durante a hospitalização das crianças constitui-se coadjuvante na terapia e pode ser percebido pelos pais ou responsáveis? O que representa brincar para os pequenos e para os adultos em uma unidade pediátrica hospitalar?

\section{OBJETIVO}

Compreender como pais e responsáveis percebem a brinquedoteca na recuperação de suas crianças hospitalizadas.

\section{METODOLOGIA}

Pesquisa exploratória descritiva com abordagem qualitativa. A perspectiva qualitativa cada vez mais tem sido reconhecida e utilizada na produção de conhecimento que analisa as várias faces e dimensões de fenômenos complexos da experiência humana (Brandão, Ribeiro, \& Costa, 2018).

O estudo teve como cenário o Hospital Infantil João Paulo II, que integra a Fundação Hospitalar do Estado de Minas Gerais, Brasil. Assiste a crianças referenciadas pelas macrorregiões estaduais e dos municípios da microrregião e da Região Metropolitana de Belo Horizonte, capital do Estado. Possui como serviços o atendimento às crianças em situação de urgência, àquelas com doenças neuromusculares e infectocontagiosas, além da atenção domiciliar em algumas situações. Sua brinquedoteca constitui-se em um ambiente físico dotado de atividades e brinquedos diversos para todas as faixas etárias. 
A coleta de dados foi realizada entre junho e julho/2018. Participaram 15 pais e responsáveis pelas crianças internadas com idades entre seis meses e 12 anos e com tempo de internação que variou de quatro a 30 dias. Foram incluídos adultos maiores de 18 anos que acompanhavam os menores e que haviam frequentado a brinquedoteca em cinco ocasiões, no mínimo. Entre os critérios de exclusão estavam os pais ou responsáveis por crianças em estado crítico, com medidas de precaução e que recebiam brinquedos no quarto ou as ausentes da unidade no período da coleta de informações.

Após a identificação dos prováveis participantes os pesquisadores abordavam os adultos em momento mais adequado, explicavam o estudo, seus objetivos e proposições e solicitavam a leitura atenta, para a assinatura do Termo de Consentimento Livre e Esclarecido. Assim, as entrevistas foram agendadas e realizadas, com gravação autorizada. Havia um espaço reservado para a coleta de informações, a partir de um roteiro semiestruturado e cada entrevista durou, em média, 30 minutos. No local estavam presentes um dos pesquisadores e o pai ou responsável, identificado por código alfanumérico, que incluiu a letra "E" de entrevistado, seguida de um número inteiro para cada participante. Foram incluídos cinco avós, três pais, seis mães e uma tia. Os pesquisadores eram três alunos do último ano do Curso de Enfermagem, do sexo feminino, treinados e orientados por tres professores, sendo dois mestres e um doutor. Todas as entrevistas foram realizadas por um dos três pesquisadores alunos e, em momento algum houve retorno aos adultos que já haviam prestado seu depoimento. O roteiro continha questões sobre a percepção da brinquedoteca no tratamento das crianças e a descrição de como acontecia o processo de brincar. As questões foram validadas com os três primeiros entrevistados para avaliar necessidade de adaptações que possibilitassem maior compreensão do que se buscava por meio do roteiro estruturado e para verificar se o objetivo poderia ser alcançado. Não houve necessidade de adaptações ou alterações e, por esse motivo, tais depoimentos foram considerados parte do estudo.

Após cada encontro o pesquisador responsável pela entrevista transcrevia as informações de forma literal. Todas as transcrições foram discutidas e avaliadas em reuniões com os professores orientadores, a cada grupo de três entrevistas, até a decisão de interromper a inclusão de novos participantes, quando todos os pesquisadores concordaram com a saturação de dados e alcance do objetivo, por meio de respostas concretas às questões da investigação. A decisão foi unânime quando passou a ocorrer repetição do conteúdo expresso. 
Em seguida, houve o recorte e a aproximação do conteúdo latente dos depoimentos que emergiu sobre o objeto do estudo. Todos os pesquisadores discutiram a semelhança de conteúdos e, em seguida, nomearam os grupos de frases e expressões mais incisivas, quando foi possível a construção de categorias temáticas representativas que possibilitariam maior entendimento e discussão dos resultados. Essa fase utilizou o referencial de Bardin. Trata-se de um conjunto de técnicas que decompõem as comunicações e que utiliza procedimentos sistemáticos e objetivos para descrever o conteúdo das informações. Procura conhecer aquilo que está por trás das palavras, sobre as quais se debruça (Bardin, 2011).

O estudo seguiu orientações da Resolução MS 466/2012 (Brasil, 2012), que trata de pesquisas com seres humanos e foi submetido e aprovado pelos Comitês de Ética em Pesquisa (CEP) da Faculdade Ciências Médicas de Minas Gerais e do Hospital Infentil João Paulo II, sob os pareceres de $\mathrm{n}^{0} \mathbf{2} .440 .198$ e 2.538.248, respectivamente.

\section{RESULTADOS}

Os participantes foram 15 pais ou responsáveis pelas crianças e os depoimentos foram organizados em três categorias, nomeadas após a análise do conteúdo latente: "O tempo de hospitalização sendo ocupado; "O vínculo criado pelas brincadeiras entre os responsáveis e as crianças"; e "O brincar como recurso terapêutico".

\subsection{O tempo de hospitalização sendo ocupado}

Os relatos enfatizaram a importância do local de brincar, pois as crianças anseiam por estar ali e permanecem felizes, por longos períodos de tempo que preenchem a lacuna gerada pela internação:

"Amanhece o dia e minha neta já chama para ir à brinquedoteca. Se não fosse isso, as crianças ficariam presas aqui, sem ter o que fazer." (E1)

"Depois que meu filho saiu da soroterapia e passou a frequentar a brinquedoteca, ficou mais alegre. Quer ficar lá o dia inteiro, pois gosta muito de vídeogame, quebra-cabeça, ele gosta de tudo." (E6)

A ociosidade, a distância da casa e o clima dos hospitais são amenizados, segundo os adultos:

"No hospital o local de brincar é o máximo, por ocupar e distrair o menino. Ele está ótimo! A brinquedoteca é uma distração." (E3) 
"Fora de casa a criança fica inquieta, tem que ter uma distração. Televisão ajuda, mas o espaço de brincar eu nunca vi em lugar algum. Muito bom." (E8).

"Cada dia é diferente: artesanato, contação de história, e tudo faz o tempo passar mais rápido." (E5)

Para os pais e responsáveis a brinquedoteca hospitalar é um espaço de alegria e distração, além de ocupar o tempo da criança. Como os adultos estão sempre juntos, fortalece os laços familiares.

\subsection{0 vínculo criado pelas brincadeiras entre os responsáveis e as crianças}

Ao brincar com os pequenos, a aproximação gerada pela brinquedoteca, foi assim revelada:

"Acho muito importante brincar com ele (referindo-se ao filho). Ele pode contar comigo." (E13)

"Gosto de brincar com ele (referindo-se ao neto), porque fica muito alegre, virou outra pessoa." (E12)

Brincar permite maior interação dos pais e responsáveis e, ainda, segundo os entrevistados, auxilia no desenvolvimento, pelo convívio com outras crianças:

"Acho importante participar de perto, porque vejo como está o seu desenvolvimento." (E4)

"Depois que milha filha veio brincar ela ficou mais envolvida, mais esperta, convive com as pessoas. Assim, a criança desenvolve mais pelo fato de conviver com outras crianças." (E9)

A brinquedoteca permite, além da interação, lembranças da própria infância dos adultos:

"Qualquer pai que teve infância e ao ver o espaço já vira criança, é importante brincar com ela. Eu acho que os pais devem participar, você ensina e a criança também nos ensina (se emociona)." (E8)

$\mathrm{Na}$ internação infantil brincar é fundamental para passar o tempo, distrair os pequenos e promover a aproximação entre os adultos e suas crianças. Os benefícios advindos relacionam-se à adaptação ao ambiente hospitalar, aderência ao tratamento e recuperação da saúde.

\subsection{O brincar como recurso terapêutico}

Para os adultos, brincar reduz a irritabilidade dos pequenos e a intolerância ao ambiente hospitalar:

"Acho a brinquedoteca uma ideia boa, reduz o estresse e a vontade de voltar para casa." (E13)

"Mesmo sentindo dor, ela (referindo-se à neta) vem para cá e, em instantes, já está melhor." (E11) 
"O comportamento de meu filho estava horroroso, ele batia em tudo e em todos, queria ir embora, gritva e acordava as pessoas de madrugada. Agora, depois que começou a brincar, está muito tranquilo." (E7)

Os participantes relacionam a brinquedoteca ao tratamento e à recuperação mais rápida da criança. Citam redução no uso de medicamentos, estabilização de marcadores clínicos e melhora no humor:

"Ele era nervoso e agitado. Agora está mais calmo. Também tem diabetes, a glicose estava muito alta e ficar brincando lá, tem deixado a glicemia na faixa dos 100, 120." (E2)

"Você acha que meu neto parava aqui, no hospital? Não, era agitado, tomava três calmantes, mas agora diminuiu." (E12)

De alguma forma, a brinquedoteca reduz as tensões provocadas pela internação e promove a adesão das crianças ao tratamento:

"Ontem meu filho melhorou e interagiu com as outras crianças. Antes só ficava deitado. Agora como ele gasta as energias na brinquedoteca, dorme a noite toda." (E7)

"Vejo que é um estimulo interessante. Minha menina chegou e já esqueceu da dor (risos). O ambiente influencia e ela reage melhor ao tratamento." (E14)

$\mathrm{Na}$ visão dos pais e acompanhantes a internação hospitalar de suas crianças é um processo doloroso para eles e para os pequenos. Afirmam que a brinquedoteca é um espaço para distração e que favorece a sua aproximação com as crianças. Além disso, os depoimentos ressaltaram a percepção da visão terapêutica do lúdico, na melhoria das relações, aceitação do tratamento e evolução favorável.

\section{DISCUSSÃO}

A brinquedoteca foi referida pelos pais ou responsáveis como um local de distração que descaracteriza o ambiente hospitalar. Trata-se de um espaço importante para crianças hospitalizadas por ser colorido e alegre e onde podem vivenciar o mundo da imaginação e da criatividade (Oliveira, Silva, \& Fantacini, 2016). Durante o tratamento o lúdico traz benefícios e contribui como um motivador para trazer de volta a autoestima dos pequenos ao mudar o foco sobre a doença e a hospitalização (Diogo, 2016; Rockembach, Espinosa, Cecagno, Thumé, \& Soares, 2017), como evidenciado nos depoimentos.

A maioria dos pais ou responsáveis enalteceu a alegria e a felicidade das crianças ao frequentar o espaço de brincar. A literatura reforça o achado ao defender que o lúdico nos hospitais potencializa o processo de adaptação pela alegria, descontração e bem estar 
emocional e comportamental em um ambiente mais agradável (Paula Marques, Garcia, Andres, Luz, Rocha, \& Souza, 2016; Jiang, 2020). Além do acolhimento, concorre para a segurança, ao diminuir a ansiedade e angústia geradas pela ameaça do desconhecido, o medo e o estresse recorrente. A partir do brinquedo e da rotina de brincar os pequenos podem elaborar seus sentimentos, enquanto hospitalizados (Diogo, 2016; Silva, Pizzoli, Amorim, Pinheiros, Romanini, Silva,... Alves, 2016; Nurwulansari, Ashar, Huriati, \& Syarif, 2019), o que foi percebido pelos pais e responsáveis no formato de adesão ao tratamento, tranquilidade durante a hospitalização e mais alegria.

O aspecto "encantador" do espaço de brincar, com ênfase nos relacionamentos construídos, tão evidenciado nos depoimentos, traduziu-se no desejo de maior tempo de permanência na brinquedoteca. Um estudo sobre o lúdico no cuidado à criança e adolescente com câncer, na perspectiva da equipe de Enfermagem, afirma que o fato de uma permanência maior nos quartos, onde há apenas adultos, acarreta sentimento de solidão. No entanto, o brincar com outras crianças promove interação e aproximação com seus pares (Paula Marques, Garcia, Andres, Luz, Rocha, \& Souza, 2016). Não deixa de ser espaço protetor que melhora o humor e ameniza o sofrimento para maior entendimento do processo de adoecimento pelas informações e elaboração de significados (Silva, Pizzoli, Amorim, Pinheiros, Romanini, Silva,... Alves, 2016; Lucietto, Lima, Gleriano, Justi, Silva, \& Borges, 2018; Gillard, 2019).

Percebeu-se ainda que a hospitalização das crianças se apresenta de forma desafiadora tanto para os pequenos, quanto para seus familiares, que necessitam alterar a rotina para acompanhar seus filhos durante o processo de internação. A brinquedoteca demostra sua importância como um espaço prazeroso, que permite o fortalecimento de vínculos ali cultivados, a partir de momentos descontraídos advindos das atividades e brincadeiras, risos e troca de atenção e carinho.

Ademais, emergiu a influência do lúdico para os adultos, diante da possibilidade de dividirem espaço e brincadeiras. Vários depoentes remeteram a lembranças do tempo em que, também, foram crianças. O espaço lúdico e terapêutico, de fato, permite uma aproximação entre pais e filhos, ao retirar, mesmo que, momentaneamente, o foco da doença e possuir várias representações. A criança se diverte, ao mesmo tempo que enfrenta e se adapta à vivência e não deixa de ser também um local de formação de cidadania (Nascimento, Madeira, Costa, Julião, \& Amorim, 2016; Paula Marques, Garcia, Andres, Luz, Rocha, \& Souza, 2016). Possibilita que o acompanhante esteja mais próximo da criança, atento às 
suas necessidades e isso o favorece para que ambos enfrentem juntos o processo (Lucietto, Lima, Gleriano, Justi, Silva, \& Borges, 2018). Ao proporcionar maior interação, a partir do brincar, evidenciou-se maior atenção dos adultos ao desenvolvimento infantil e à aquisição de competências como viver em sociedade, respeitar os outros e lidar com sentimentos.

De fato, pesquisas sobre os aspectos pedagógicos dos espaços para brincar, em hospitais, para além de ferramenta que auxilia no cuidado, reforçam os achados em relação à percepção de que a criança aprende e ensina os adultos, além de favorecer a aceitação dos procedimentos intrínsecos ao tratamento.

Uma delas aponta que brincar facilita a adaptação ao novo dia a dia e a compreensão do que se passa na fase vivida, para que a criança se torne autor de sua história (Lucietto, Lima, Gleriano, Justi, Silva, \& Borges, 2018). A segunda, defende que a brinquedoteca hospitalar possibilita a interação do menor entre momentos de alegria e prazer e, até mesmo, o esquecimento da razão de estar ali (Oliveira, Silva, \& Fantacini, 2016).

Ademais, os adultos perceberam a relevância da brinquedoteca, em uma instituição hospitalar infantil, por proporcionar benefícios que permeiam a aderência à terapia necessária, com maior tranquilidade das crianças. A hipótese formulada de benefícios terapêuticos percebidos pelos adultos em relação a suas crianças, pode ser comprovada, o que significa redução dos impactos da hospitalização (Nurwulansari, Ashar, Huriati, \& Syarif, 2019). Na literatura, os achados enaltecem a importância do brincar na internação pediátrica ou, especificamente, antes e durante os procedimentos hospitalares necessários e concluiram que as atividades lúdicas proporcionam bem-estar e ajudam na recuperação (Jiang, 2020). As crianças com medo, silenciosas e não cooperativas alteraram comportamentos, o que sugere espontaneidade para cooperar e melhor aceitar o tratamento. Assim, a brinquedoteca favorece interações sociais, reduzem a dor e concorrem para a melhora clínica (Diogo, 2016; Oliveira, Silva, \& Fantacini, 2016; Silva, Pizzoli, Amorim, Pinheiros, Romanini, Silva,...Alves, 2016; Rockembach, Espinosa, Cecagno, Thumé, \& Soares, 2017; Gillard, 2019).

Isso posto, o estudo permitiu compreender a percepção dos pais e responsáveis sobre o papel da brinquedoteca na recuperação de sua criança em uma unidade pediátrica hospitalar. A análise em profundidade dos depoimentos, pelo referencial metodológico e sua comparação com a literatura atual sobre o tema foram fundamentais para problematizar e apontar caminhos que, segundo Brandão, Ribeiro e Costa (2018), permitem conhecer, 
perceber e intervir sobre questões identificadas. Pode-se afirmar que somente a abordagem qualitativa, livre das amarras numéricas, é capaz de analisar o subjetivo, dar voz e potencializar o exercício dessa cidadania.

A pesquisa possui limitações por ter sido desenvolvida em apenas uma instituição hospitalar, que poderia representar apenas a realidade local, apesar de ser considerada serviço de referência no Estado para atendimento pediátrico. Porém, mais que generalizações, buscou-se o despertar para novos caminhos que qualifiquem o cuidado pediátrico. Outros estudos que abordem as demais facetas assistenciais devem se constituir. Por exemplo, a própria visão da criança, ou mesmo, dos profissionais de saúde, no desempenho de suas atividades, na presença ou ausência de brinquedotecas.

\section{CONCLUSÕES}

A hospitalização pediátrica é um processo complexo, pois a criança é retirada de seu ambiente habitual e inserida em um local desconhecido, distante de seus objetos, de sua rotina e de seus hábitos. O menor tende a apresentar dificuldade na adesão ao tratamento hospitalar e, tal resistência, se manifesta a partir de comportamentos, como irritabilidade, choro e negação aos procedimentos.

Mecanismos como a ludoterapia, são capazes de amenizar o sofrimento advindo da internação, ao aproximar a criança do contexto ao qual ela já possui identificação. A partir do ato de brincar, pais e responsáveis percebem a redução das tensões em suas crianças hospitalizadas, o aumento de sua autoestima e sensação de segurança, o que contribui para sua recuperação.

A abordagem qualitativa no estudo permitiu concluir que a sala de brincar e as atividades ali desenvolvidas são terapêuticas, assim como está sustentado pela literatura. Os benefícios elencados envolvem desde a ocupação do tempo e diversão, até o fortalecimento de dos laços afetivos entre os pequenos e seus familiares.

A contribuição na adaptação ao ambiente hospitalar e na aderência ao tratamento é visível, por pais e acompanhantes, para a recuperação dos pequenos.

Gestores e administradores poderiam introduzir, otimizar e valorizar o espaço de brincar, com vistas à redução do tempo de internação, maior rotatividade de leitos, redução da 
angústia de pais, responsáveis e das próprias crianças ao vivenciar a experiência da hospitalização.

\section{REFERÊNCIAS}

Araújo, R. A. S., Ribeiro, M. C. O., Sobral, A. L. O., Silva, F. A., \& Faro, A. (2017). Uso de atividades lúdicas no processo de humanização em ambiente hospitalar pediátrico: relato de experiência. Interfaces - Revista de $\begin{array}{llr}\text { Extensão da UFMG, } & \text { 5(1), } & 166-172 .\end{array}$ https://www.ufmg.br/proex/revistainterfaces/index.php/IREXT/article/view/124

Bardin, L. (2011). Análise de conteúdo. São Paulo: Edições 70.

Brandão, C., Ribeiro, J., \& Costa, A. P. (2018). Investigação qualitativa: em que ponto estamos? Ciência \& Saúde Coletiva, 23(1), 4. https://doi.org/10.1590/1413-81232018231.27992017

Brasil. (2012). Ministério da Saúde. Conselho Nacional de Saúde. Resolução no 466, de 12 de dezembro de 2012. Aprova diretrizes e normas regulamentadoras de pesquisas envolvendo seres humanos. http://conselho.saude.gov.br/resolucoes/2012/Reso466.pdf

Conceição, L. S. (2016). A influência do lúdico no cuidado e tratamento de crianças hospitalizadas. Psicologia. PT. O portal dos psicólogos. https://www.psicologia.pt/artigos/textos/A1002.pdf

Diogo, J. S. (2016). Brinquedoteca hospitalar: a importância do brincar durante o processo de tratamento infantil na internação. Psicologia. PT. O Portal dos Psicólogos. https://www.psicologia.pt/artigos/ver_artigo.php?brinquedoteca-hospitalar-a-importancia-do-brincardurante-o-processo-de-tratamento-infantil-na-internacao\&codigo=A1010

Gabatz, R. I. B., Terra, A. P., Couto, G. R., Milbrath, V. M., \& Schwartz, E. (2017). Hospitalization in the child's perspective: an integrative review. Revista de enfermagem, 11(2), 703-711. https://doi.org/105205/19818963-v11i2a11988p703-711-2017

Gillard, A. (2019). Outcomes of a hospital-based recreation program. Children and Youth Services Review, 96, 79-83. https://doi.org/10.1016/j.childyouth.2018.11.037

Jiang, S. (2020). Positive Distractions and Play in the public Spaces of Pediatric Healthcare Environments: A Literature Review. HERD: Health Environments Research \& Design Journal. https://doi.org/10.1177/1937586720901707

Leite, T. M. C., Vergílio, M. S. T. G., \& Silva, E. M. (2017). Pediatric nurse's work process: a reality to be transformed. Revista da Rede de Enfermagem do Nordeste, 18(1), 26-34. https://doi.org/ 10.15253/21756783.2017000100005

Lucietto, G. C., Lima, L. T. S., Gleriano, J. S., Justi, J., Silva, R. A., \& Borges, A. P. (2018). Toy library as supporting tool in care: perception of nursing professionals. Revista Saúde e Desenvolvimento, 12(10), 2634. https://www.uninter.com/revistasaude/index.php/saudeDesenvolvimento/article/view/870

Marcolin, M., Marcolin, G. C. A., Souza, A. R., \& Barbosa, A. C. R. (2016). Implantação de uma brinquedoteca no setor pediátrico do Hospital Municipal Santana de Carandaí, Minas Gerais: um relato de experiência. Journal of Management and Primary Health Care, 7(1), 98. https://doi.org/1014295/jmphc.v7i1.424

Nascimento, R. R., Madeira, M. Z. A., Costa, M. S. A., Julião, A. M. S., \& Amorim, F. C. M. (2016). The play area as a tool in assisting the child hospitalized, about the look of the caretaker. Revista Interdisciplinar, 9(2), 2937. https://revistainterdisciplinar.uninovafapi.edu.br/index.php/revinter/article/view/885/pdf_309 
Nurwulansari, N., Ashar, M. U., Huriati, H., \& Syarif, S. (2019). The Effect of Constructive Play Therapy on Anxiety Levels of Preschool Children Due to Hospitalization. Journal of Health Science and Prevention, 3(3S), 72-78. https://doi.org/10.29080/jhsp.v3i3S.282

Oliveira, E. F., Silva, V. M., \& Fantacini, R. A. F. (2016). Hospitals pedagogy: the toy library environments in hospital. Research, Society and Development, 1(1), 88-104. https://doi.org/ 10.17648/rsd-v1i1.6

Paula Marques, E., Garcia, T. M. B., Andres, J. C., Luz, J. H., Rocha, P. K., \& Souza, S. (2016). Playful activities in health care for children and adolescents with cancer: the perspectives of the nursing staff. Escola Anna Nery, 20(3), e20160073. https://doi.org/10.5935/1414-8145.20160073

Pinto, M. B., Andrade, L. D. F., Medeiros, A. P. G., Santos, G. L. O., Queiroz, R., \& Jales, R. D. (2015). Atividade Lúdica e sua importância na Hospitalização infantil: uma revisão integrativa. Revista da Universidade Vale do Rio Verde, 13(2), 298-312. http://dx.doi.org/10.5892/ruvrd.v13i1.2292

Rockembach, J. A., Espinosa, T. A., Cecagno, D., Thumé, E., \& Soares, D. C. (2017). Inserção do lúdico como facilitador da hospitalização na infância: percepção dos pais. Journal of Nursing and Health, 7(2), 117-126. https://doi.org/10.15210/jonah.v7i2.7646

Silva, D. F., \& Brandão, E. C. (2017). As práticas lúdicas no cotidiano do cuidar em enfermagem pediátrica. Revista de Enfermagem da Faciplac, 1(1), 1-2. file:///C:/Users/Usu\%C3\%A1rio/Downloads/266-565-1SM.pdf

Silva, J. R. S., Pizzoli, L. M., Amorim, A. R., Pinheiros, F. T., Romanini, G. C., Silva, J. G.,... Alves, S. S. (2016). Using Therapeutic Toys to Facilitate Venipuncture Procedure in Preschool Children. Pediatric Nursing, 42(2), 61-68. https://www.ncbi.nlm.nih.gov/pubmed/27254974 